\title{
LOS DIPTONGOS LARGOS EN LATÍN Y EN ITÁLICO
}

\begin{abstract}
Against the doctrine commonly accepted, the author states that there are not definite traces of differential treatments of the long diphthongs neither in Latin nor in any other Italic language.
\end{abstract}

Es doctrina común que los diptongos indoeuropeos de primer elemento largo no presentan en las lenguas itálicas ningún resultado diferencial respecto a los breves en posición inicial e interior. En cambio, en posición final presentarían resultados diferentes de los breves, especialmente en latín, y más esporádicamente en alguna lengua itálica ${ }^{1}$.

Los únicos representantes del tal tratamiento diferencial se encontrarían en el dativo de singular temático en - $* \bar{o} i$ y en ciertas formas casuales de los femeninos en $-\bar{a}$.

\section{El diptongo largo $-* \bar{o} \mathrm{i}$}

En el dativo de plural -*ōis sufriría una temprana abreviación en -*ois que lo confundiría con el diptongo /oi/ breve de sílaba final, cuyo resultado fonético ulterior compartiría. Esta confusión es común a todas las lenguas itálicas: lat. -eis (-īs), osco -úis (|ois/), umbro - $e^{3}$ (de -*ois), etc. ${ }^{2}$

En dativo de singular, el diptongo -*ōi presentaría un doble tratamiento: $-\bar{o} / o i$, generalmente explicado como doblete de sandhi. Las lenguas que

A. Ernout 1903-1906, p. 322; M. Leumann 1963, pp. $67-68$ y 90; 1977, p. 62.

2 M. Leumann 1963, p. 280; 1977, pp. 416, 421 y 428-29; R. von Planta 1897, pp. 124-125; etc. Respecto a las formas del marso esos, aisos cf. M. Durante 1978, pp. 804 y 807 . 
tienen -oi o su resultado fonético, también aquí lo confunden con el correspondiente diptongo breve: osco $-u ́ i(\mid o i /)$, umbro $-e^{3}$ (de -*oi), falisco $-o i^{4}$, véneto $-o i^{5}$.

Otras lenguas, en cambio, tienen - $\bar{o}$, principalmente representado en latín y vestino ${ }^{6}$. A su vez, hay una serie de testimonios esporádicos latinos de dativo temático en -oi de fecha arcaica: Numasioi, Duenoi, Populoi Romanoi, cameloi, caproi. Aparte de la escasa solidez de estos testimonios ${ }^{7}$, nada prueba que se trate de $|\bar{o} i|$ conservado con su cantidad larga. Si esas formas han sido reales en latín, se trata sin lugar a dudas de -oi, tal como es el dativo temático en las demás lenguas itálicas. Como hizo ver Walde ${ }^{8}$, ningún hecho garantiza que $-\bar{o}$ clásico proceda del -oi arcaico mediante un proceso fonético ocurrido al principio de la tradición escrita latina. De hecho, la mayoría de los lingüistas considera que -oi (arcaico) y -ō (clásico) son dos variantes de sandhi producidas en época prehistórica a partir de la forma originaria -*ōo $i$ que, como tal, deja de existir.

Tampoco la cronología relativa exige que $-o i$ arcaico sea $-* \bar{o} i$, puesto que los diptongos -oi originarios aparecen inalterados en los primeros documentos latinos $^{9}$.

En resumen, el único resultado diferencial, la única huella efectiva del diptongo largo -*ōo sería la forma del dativo de singular temático $-\bar{o}$.

\section{El diptongo largo $-* \bar{a} i$}

También este diptongo aparece confundido con el correspondiente breve en el dativo plural: lat. -is, osco -ais, umbro -es ${ }^{10}$. Igualmente confundido con el breve aparece en el dativo de singular del osco -ai, umbro $-e$, marrucino $-a i$, marso $-e$, volsco $-e^{11}$.

Pero junto a esos casos de confusión, existen varios otros tenidos por tratamientos diferenciales:

${ }^{3}$ R. von Planta 1897, pp. 109-111.

4 G. Giacomelli 1978, p. 517

5 A. Prosdocimi 1978, p. 332

- Sobre el testimonio esporádico de $-\bar{o}$ en otras lenguas itálicas cf. F. Villar 1985 , pp. $32-35$.

7 Sobre el valor de estos testimonios véase también F. Villar 1985, p. 32, n. 21. Resumiendo diré que Numasioi es sospechoso de ser falso como la fibula en que aparece inscrito (cf. recientemente M. Guarducci 1980). Duenoi es de lectura e interpretación dudosa. Los restantes son testimonios indirectos de Mario Victorino.

${ }^{8}$ A. Walde 1916, pp. 197-99; cf. 1917, pp. 38-39.

${ }^{9}$ Cf. quoi en $C I L \mathrm{I}^{2} 1$ y qoi en $C I L \mathrm{I}^{2} 4$.

10 R. von Planta 1897, p. 99.

11 R. von Planta 1897, pp. 88-92. 
a) Dativo de singular latino en -ae. En efecto, el diptongo breve -*ai aparecería desde el principio de la tradición como -ei (luego $-\bar{l})$, mientras que el antiguo diptongo largo aparecería desde el principio de la tradición como -ai (luego-ae).

b) Dativo singular latino en - $a$. Este dativo epigráfico (Diana, Loucina, etc.) sería el paralelo del temático $-\bar{o}$ y tendría su mismo origen en sandhi.

c) Nominativo de plural en -a: Matrona (CIL I $\mathrm{I}^{2} 378$ y 379). El nominativo plural femenino latino habría sido $-* \bar{a} i^{12}$ y en este ejemplo presentaría el mismo tratamiento que los dativos de singular Diana, Loucina, etc. ${ }^{13}$.

d) Dativo-ablativo de plural: (Deuas Corniscas, Anabestas, Aestimias). Se trataría de un caso más de tratamiento $-\bar{a}$ del diptongo -*āi al igual que en Diana, Loucina de los que sería la contrapartida en plural ${ }^{14}$.

\section{Critica de los datos}

Como hemos visto, las huellas de los diptongos largos en las lenguas itálicas se reducirían a: 1) Matrona, 2) Deuas Corniscas, Anabestas, Aestimias, 3) Dativo temático en $-\bar{o}, 4)$ Dativo femenino en $-\bar{a}$ y 5) Tratamiento -ae. Examinemos ahora el valor de este material.

\section{Matrona}

Se encuentra en dos inscripciones de Pisauro (CIL $\mathrm{I}^{2} 378$ y 379$)$. La primera de ellas presenta una fractura de la piedra justamente tras la $-A$, lo que hace inseguro que se trate realmente de una forma terminada así. Blümel ${ }^{15}$ estima que hay espacio suficiente para $S$ o para $I$, lo que nos daría Matronas o Matronai. En cualquiera de esos casos CIL $\mathrm{I}^{2} 378$ causa baja como posible testigo de un tratamiento hipotético $-* \bar{a} i>-\bar{a}$.

Nos queda únicamente el ejemplo de $C I L \mathrm{I}^{2} 379$. Aparte de una interpretación como nominativo de singular propuesta por Kajanto ${ }^{16}$, si se

\footnotetext{
12 La cantidad larga de ese diptongo es una hipótesis de Solmsen 1894, pp. 242-43. Véase una crítica de la misma en F. Villar (en prensa).

13 Se trata de una hipótesis de A. Ernout 1903-1906, p. 324.

14 Esa explicación es también una hipótesis de A. Ernout 1903-1906, p. 322 ss.

15 W. Blümel 1972, p. 132, n. 136.

16 I. Kajanto 1967, pp. 72-77.
} 
mantiene la generalmente admitida como nominativo de plural, la opinión generalizada es la de que se trata de un nominativo de plural en $-\bar{a}(s)^{17}$. La hipótesis de que se trate de $-* \bar{a} i>-\bar{a}$ es de Ernout, y su propio autor se inclina por $-\bar{a}(s)$ en una obra posterior ${ }^{18}$.

\section{Deuas Corniscas, Anabestas, Aestimias}

Deuas Corniscas se encuentra en CIL I ${ }^{2}$ 975; Anabestas en CIL I ${ }^{2} 969$; y Aestimias en Festo 24, 15. La interpretación genética de estas formas ha sido objeto de prolongada polémica. Las hipótesis emitidas al respecto son:

a) Se trata de un antiguo locativo de plural -*āsu con pérdida de la $-u$ final (Sommer, Pisani) ${ }^{19}$.

b) Se trata de un osquismo, partiendo de -*ābhos $>-* \bar{a} f s>-\bar{a} s$ (Pisani) $^{20}$.

c) Se trata de un dativo de plural en -*āis con pérdida del segundo elemento del diptongo (Ernout) ${ }^{21}$.

Esas tres propuestas coinciden en intrepretar sintácticamente Deuas Corniscas como un dativo de plural. Frente a ello:

d) Se trataría de un genitivo de singular en -ās (Ribezzo, Blü$\mathrm{mel})^{22}$.

Los argumentos contra el análisis de Deuas Corniscas como genitivo de singular han sido, en general, que el sintagma Deuas Corniscas sacrum exige entenderlo como dativo; y que no puede ser singular porque Corniscarum Diuarum locus (Festo 56,14) demuestra que se trataba de una divinidad plural.

Blümel ${ }^{23}$ ha argumentado sólidamente contra esas dificultades y ha demostrado que, a pesar de ellas, se trata de un genitivo de singular. En efecto, no necesita ser plural, ya que existe un testimonio seguro de esa misma divinidad en singular: Cornicei $\left(C I L I^{2}\right.$ 976). Tampoco necesita ser

17 M. Leumann 1963, p. 276 (con bibliografia); 1977, pp. 420-21; V. Pisani 1962, p. 157 ; L. R. Palmer 1954 , p. 241 ; etc.

18 A. Ernout 1953, p. 22; pero cf. 1966, p. 42 donde vacila entre ambas posibilidades.

19 F. Sommer 1948, p. 332; V. Pisani 1962, p. 153.

20 V. Pisani 1960 , p. 242.

21 A. Ernout 1903-1906, p. 322 ss.

22 F. Ribezzo 1920; W. Blümel 1972, pp. 48-49.

23 W. Blümel 1972, pp. 48-49. 
dativo, ya que puede ser genitivo: con sacrum ambas construcciones se dan y no son exactamente sinónimas ${ }^{24}$.

En lo que a Anabestas se refiere, hay que señalar que carece de contexto, ya que la inscripción consiste en esa sola palabra. Como hace ver Blümel, si hay que suplir sacrum, revierte a la interpretación de Deuas Corniscas.

A la convincente argumentación de Blümel quiero añadir una reflexión sobre la improbabilidad de que estas formas procedan de -*äis. El tratamiento latino ordinario en -eis (luego $-\bar{l} s$ ) es idéntico al de los diptongos breves -*ai y distinto al de los largos (-ae). Tradicionalmente ello se ha explicado por una tempranísima abreviación de -*āis en -*ais que ha permitido su pronta confusión con los diptongos /ai/ breves de sílaba final cuya suerte ha compartido.

Desde el marco de la explicación tradicional de estos problemas en que se mueve Ernout, su explicación de estas formas en $-\bar{a} s$ como dativos de plural a partir de $-* a \bar{a}$ is tiene dos severos inconvenientes:

1) Exige la conservación de -*āis con su cantidad larga hasta el momento en que todos los diptongos largos -*ōo y -*āi generan los dobletes $-\bar{o} / o i$ y $-\bar{a} /-\bar{a} i$. En ese momento se habría producido paralelamente $-\bar{a} s /-a i s$ a partir de -*āis. Ello, en la versión fonética en que se mueve Ernout, implica que -*ais surgido de -*āis no habría tenido tiempo de confundirse con /-ai/ antiguo ni de seguir su tratamiento, como no lo tuvo - siempre según esa versión- en el dativo de singular donde no da $-e i>-\bar{l}$ sino -ae. Es decir, esperaríamos entonces un dativo de plural latino -*aes y no -īs. La cronología relativa, pues, se opone a esta interpretación.

2) La explicación de Ernout no tiene justificación fonética. En efecto, en el caso de los dativos de singular los dobletes se justifican tradicionalmente como variantes de sandhi en posición antevocálica/anteconsonántica. Ahora bien, ello no es aplicable al dativo de plural cuya $-s$ final ponía al diptongo largo al abrigo de esas alternativas.

En consecuencia, no existe probabilidad de que estas formas en $-\bar{a} s$, caso de ser realmente dativos de plural, procedan fonéticamente de $-* \bar{a} i s$.

\section{El dativo temático en $-\overline{\mathrm{o}}$}

Hoy se acepta ampliamente que esta modalidad de dativo temático es un hecho gramatical que se encuentra ampliamente difundido en la fami-

24 G. Camporeale 1967, p. 69 ss. 
lia indoeuropea. En trabajos anteriores ${ }^{25}$ he hecho ver que se encuentra en celta, germánico, báltico, indio, iranio y hetita. Y creo haber demostrado que entre $-* \bar{o} i$ y $-* \bar{o}$ no existe relación fonética. Por el contrario, $-* \bar{o}$ es la forma más antigua y - $* \bar{o} i$, en las lenguas en que se encuentra, es una innovación analógica a partir de todos los demás dativos que tienen $/ i /$.

En consecuencia, el dativo temático en $-\bar{o}$ causa baja como testigo de un tratamiento diferencial de los diptongos largos.

\section{Dativo femenino en - $\bar{a}$}

La limitación de esta forma al latín, donde hay $-\bar{o}$ en los masculinos, y la génesis no fonética de esta última me han llevado en otro trabajo ${ }^{26}$ a concluir que el dativo en $-\bar{a}$ es una imitación analógica de $-\bar{o}$, debida a la fuerte tendencia a equiparar las desinencias de los masculinos y los femeninos en latín. Alli explico las circunstancias que llevaron a esa innovación. Por consiguiente, el dativo en $-\bar{a}$ tampoco es un testigo del tratamiento diferencial de los diptongos largos.

\section{Tratamiento -ae en el dativo femenino}

Tradicionalmente se suponía que en posición final $-*$ ai daba $-\bar{\imath}$, mientras que $-* \bar{a} i$ daba $-a e$. En otro trabajo ${ }^{27}$ he puesto de manifiesto que $-\bar{l}$ se encuentra como resultado tanto de diptongos breves (primera persona de singular del perfecto) como de diptongos largos (dativo de plural femenino). Y que, por su parte, -ae se da igualmente con diptongos largos (dativo de singular) y breves (nominativo de plural [cf. gr. - $\alpha$ l], nae, prae, etc.).

El tratamiento diferencial $-\bar{l} /$-ae no depende, pues, de la cantidad etimológica del diptongo, sino del carácter cerrado o abierto de la sílaba. En el perfecto, de acuerdo con ello, habría que tener -*ae, $-^{*}$ istae, $-\bar{l} t$, pero todas las personas se han nivelado analógicamente en $-\bar{l}$ a partir de la tercera.

En consecuencia, el tratamiento - ae tampoco es un testimonio diferencial de los diptongos largos.

\footnotetext{
25 F. Villar 1981, pp. 147-49, 164-68, 196 ss.; 1985.

26 F.Villar 1986.

27 F. Villar (en prensa).
} 


\section{CONCLUSIÓN}

Como conclusión de lo expuesto hasta ahora se impone el hecho de que ni en latín ni en itálico hay huella alguna de tratamientos diferenciales de los diptongos largos. Todos los diptongos largos indoeuropeos que fueran transmitidos como tales al latín y a las restantes lenguas itálicas se abreviaron en fechas prehistóricas, a tiempo de confundirse siempre y en todas posiciones con los correspondientes diptongos breves y compartir su ulterior suerte fonética.

Expresado en otros términos: por el testimonio del latín y de las lenguas itálicas no se lograría detectar la existencia de dos tipos de diptongo en indoeuropeo (de primer elemento breve/de primer elemento largo).

F. VILLAR

\section{BIBLIOGRAFÍA}

Blümel, W. 1972: Untersuchungen zu Lautsystem und Morphologie des vorklassischen Lateins, Munich.

Camporeale, G. 1967: «Note sulle dediche umbre a Cupra da Colfiorito», RAL (serie $\left.8 . .^{a}\right) 22$, pp. 65-72.

Durante, M. 1978: «I dialetti medioitalici», Popoli e Civiltà dell'Italia Antica, VII. Lingue e Dialetti, Roma, pp. 789-824.

Ernout, A. 1903-1906: «Le parler de Préneste d'après les inscriptions», MSL 13, pp. 293-349.

-1953: Morphologie Historique du latin (3." ed.), París.

1966: Recueil de textes latins archaïques (2.a ed.), Paris.

Giacomelli, G. 1978: «Il falisco», Popoli e Civiltà dell'ttalia Antica, VII. Lingue e Dialetti, Roma, pp. 505-42.

Guarducci, M. 1980: «La cosidetta fibula Praenestina», MAL 24, pp. 413-574.

Kajanto, I. 1967: "Contribution to Latin morphology», Arctos 5, pp. 67-77.

Leumann, M. 1963: Lateinische Laut- und Formenlehre, Munich.

-1977: Lateinische Laut- und Formenlehre, Munich.

Palmer, L.R. 1954; The Latin Language, Londres.

Pisani, V. 1962; Grammatica Latina (3." ed.), Turín.

von Planta, R. 1897: Grammatik der oskisch-umbrische Dialekte, II, Estrasburgo.

Prosdocimi, A. 1978: «Il venetico», Popoli e Civiltà dell'Italia Antica, VII. Lingue e dialetti, Roma, pp. 257-380.

Ribezzo, F. 1920: «Esistenza e uso del genitivo dedicatorio nel latino preletterario», RIGI 4, pp. 77-82.

Solmsen, F. 1894: «Der Infinitiv Praesentis Activi und die $i$-Diphthonge in wortschliessenden Silben im lateinischen", IF 4, pp. 240-52.

Sommer, F. 1948: Handbuch der lateinischen Laut- und Formenlehre (3." ed.), Heidelberg. 
Villar, F. 1981: Dativo y Locativo, Salamanca.

- 1985: «El dativo temático indoeuropeo», Symbolae Mitxelena, Vitoria, pp. 31-48.

- 1986: «El dativo latino epigráfico en -ā», EMERITA 54, pp. 45-62.

-(en prensa): "The Latin Diphthongs - ai, - $\bar{a} i$ in Final Syllables", IF.

Walde, A. 1916: "Die italischen Sprachen", Geschichte der indogermanischen Sprachwissenschaft II 1, pp. 127-230.

- 1917: Über ältesten sprachliche Beziehungen zwischen Kelten und Italikern, Innsbruck. 